\title{
Los sistemas de información geográfica como herramienta para monitorear las desigualdades de salud
}

\author{
Enrique Loyola, ${ }^{1}$ Carlos Castillo-Salgado, ${ }^{1}$ \\ Patricia Nájera-Aguilar, ${ }^{1}$ Manuel Vidaurre, ${ }^{1}$ \\ Oscar J. Mujica ${ }^{1}$ y Ramón Martínez-Piedra ${ }^{1}$
}

RESUMEN Objetivos. Mostrar la aplicación de los sistemas de información geográfica (SIG) como instrumento tecnológico para apoyar las actividades en las áreas de política sanitaria y salud pública.

Métodos. Se evaluó la relación entre la mortalidad infantil y diversos factores determinantes de carácter socioeconómico y geográfico. Al ilustrar la aplicación, se hace hincapié en la capacidad integradora de los SIG, que permite simplificar, agilizar y automatizar la evaluación epidemiológica, tomando en cuenta el análisis múltiple simultáneo de variables determinantes con diferentes niveles de agregación. La aplicación de los SIG abarcó, en este estudio, el análisis de la mortalidad infantil en tres niveles de agregación en países de las Américas entre 1995 y 2000.

Resultados. La mortalidad infantil estimada para la Región tuvo un promedio de 24,4 defunciones por 1000 nacidos vivos, pero las desigualdades observadas indican que la probabilidad de una muerte infantil es casi 20 veces mayor en los países de menos recursos que en los más prósperos. El mapeo de la mortalidad infantil a escala regional permitió identificar los países que requieren mayor atención en sus políticas y programas de salud, pero no distinguir dónde se requerían acciones más prioritarias. Un análisis de las unidades geopolíticas más pequeñas (estados y municipios) reveló importantes diferencias dentro de los países y permitió reproducir el patrón de desigualdad regional, que no se ve reflejado por el valor promedio de los indicadores a escala nacional. Al analizarse la relación entre el analfabetismo femenino y la desnutrición como factores determinantes de la mortalidad infantil en Brasil y Ecuador, se identificaron estratos sociales y epidemiológicos con distribuciones diferenciales de factores de riesgo que requieren intervenciones sanitarias adecuadas para sus respectivos perfiles socioepidemiológicos.

Conclusiones. Gracias a este tipo de análisis epidemiológico a escala local de los servicios de salud mediante el uso de los SIG, es fácil reconocer cómo se comportan un fenómeno de salud y sus factores de riesgo determinantes en un período definido. Asimismo, es posible identificar patrones en la distribución espacial de los factores de riesgo y sus posibles efectos sobre la salud. La utilización adecuada de los SIG permitirá lograr mayor eficacia y equidad en la prestación de los servicios de salud pública.

Palabras clave

\footnotetext{
1 Programa Especial de Análisis de Salud, Organización Panamericana de la Salud, Washington, D.C., EE.UU. La correspondencia debe dirigirse a: Enrique Loyola, OPS/WHO, 525 Twenty-third St., N.W., Washington, D.C. 20037, U.S.A.
}

Sistemas de información geográfica, desigualdad, mortalidad infantil, necesidades insatisfechas.
La equidad en materia de salud se considera uno de los principios básicos para el desarrollo humano y la justicia social (1). Cómo alcanzar 
niveles cada vez más altos de equidad en el campo de la salud en un mundo que es objeto de diferentes reformas sociales y económicas suscita gran preocupación entre las instancias decisorias y grandes segmentos de la población. Aunque se sigue debatiendo lo que significa la equidad en salud, la búsqueda de métodos e indicadores para medir las desigualdades en salud es también un campo en continua expansión (2).

Uno de los primeros pasos para determinar la magnitud de las inequidades en el área de la salud es la medición de las desigualdades (2). Hasta ahora, las necesidades de salud insatisfechas que afectan a diversos grupos poblacionales han sido utilizadas como medidas alternativas (proxy) para definir y monitorear las desigualdades en salud. Esta medición y el monitoreo de los problemas de salud son esenciales para la adecuada asignación y distribución, tanto social como geográfica, de los recursos y servicios de salud. Se reconoce que la Región de las Américas tiene la mayor desigualdad social en el mundo (3). No obstante, hay poca información empírica que documente la magnitud de las desigualdades en el campo de la salud y su distribución en las Américas. Entre las dificultades que han enfrentado hasta ahora los gerentes de salud y otras entidades decisorias para realizar estos análisis figuran la poca disponibilidad de datos específicos desagregados, la carencia de herramientas analíticas más eficientes que tomen en cuenta la dimensión geográfica, y la ausencia de guías y ejemplos basados en situaciones reales. La Organización Panamericana de la Salud (OPS) ha estado trabajando en estas diversas esferas para, por un lado, estimular la producción de datos básicos desagregados (4) y, por otro, facilitar su análisis en los países mediante el uso de nuevas tecnologías y métodos sencillos, como los que aparecen en otros artículos de esta publicación y en otras contribuciones $(5,6)$.

El objetivo principal de este artículo es describir la situación de las desigualdades en materia de salud que se observan en la Región de las Américas usando como indicador la mortalidad infantil, como una primera aproximación a la definición de necesidades insatisfechas, y definir su relación con algunos de sus factores determinantes de carácter socioeconómico mediante métodos analíticos sencillos. Se presenta una serie de análisis de distintos niveles de agregación que tratan de dar respuesta a las dificultades mencionadas anteriormente e ilustran las capacidades y potencialidades del uso de los sistemas de información geográfica (SIG) para monitorear las desigualdades en el ámbito de la salud. Un propósito adicional de este artículo es mostrar cómo se pueden simplificar los análisis complejos de la situación de salud para beneficio de las autoridades decisorias y la población en general.

\section{MATERIALES Y MÉTODOS}

Uno de los indicadores más frecuentemente utilizados para evaluar la situación de salud de un país sigue siendo la tasa de mortalidad infantil, que se expresa como el número de muertes de niños menores de un año que se producen anualmente en un área geográfica, dividido por la población de niños nacidos vivos registrados el mismo año. Las tasas nacionales medias de mortalidad infantil aquí presentadas han sido notificadas a la OPS por sus Estados Miembros durante el período de 1995-2000 (7-11), mientras que los datos del primer nivel subnacional (estados, departamentos y provincias) son los publicados por los países en sus indicadores básicos según las recomendaciones sobre estándares, y los referidos en el Informe anual del Director de la OPS del año 2000 (12). A su vez, los datos del segundo nivel subnacional (municipios y cantones) de Brasil y Ecuador fueron obtenidos

\footnotetext{
2 DataSUS es el sistema de informática del Sistema Único de Salud (SUS) de Brasil, que tiene la responsabilidad de mantener, estandarizar e integrar los datos de sus diversos sistemas.
}

de DataSUS ${ }^{2}$, por conducto del Centro Nacional de Epidemiología (CENEPI) y del INFOPLAN, respectivamente $(13,14)$. Los datos de mortalidad infantil de estos países corresponden a estimaciones demográficas hechas por dichas instituciones a partir de los datos de los censos de 1990 y de sus proyecciones con modelos de regresión tipo BrassTrussell. Paralelamente a la mortalidad infantil, se usaron algunos indicadores de determinantes socioeconómicos conocidos -en particular el analfabetismo femenino y la desnutrición- para evaluar su relación con la distribución de los niveles de riesgo a la salud en los países. En el caso de Ecuador, el analfabetismo corresponde a la población mayor de 15 años y la desnutrición está definida a partir de una desviación estándar de la talla media esperada para la edad.

Las bases de datos cartográficos a escala nacional y de primer nivel subnacional para los mapas temáticos de las Américas provienen del Atlas digital del mundo (15) y fueron editadas por la OPS ${ }^{3}$; se trata de mapas a una escala de 1:100 000 000. Las bases de datos cartográficos de segundo nivel subnacional fueron suministradas por el Instituto Brasileño de Geografía y Estadística (IBGE), por conducto del CENEPI, y por el Instituto Nacional de Estadística y Censos (INEC) de Ecuador, por mediación del INFOPLAN $(14,16)$. La escala de los mapas nacionales es de 1:25000 000 en el caso de Brasil y de 1:5000 000 en el de Ecuador, y los levantamientos corresponden a 1997 y 1995, respectivamente. La imagen de satélite utilizada corresponde al relieve continental y marino del mundo $\mathrm{y}$

\footnotetext{
La OPS no avala necesariamente la distribución

4 Eoliticogeográfica de los países. de resumir los datos en que la línea horizontal representa la mediana de la distribución de frecuencias, los extremos de la caja representan el intervalo entre cuartiles (interquartile range) y la extensión de las líneas los valores extremos. Se observan separadamente puntos que representan observaciones muy alejadas de los valores esperados (outliers).
} 


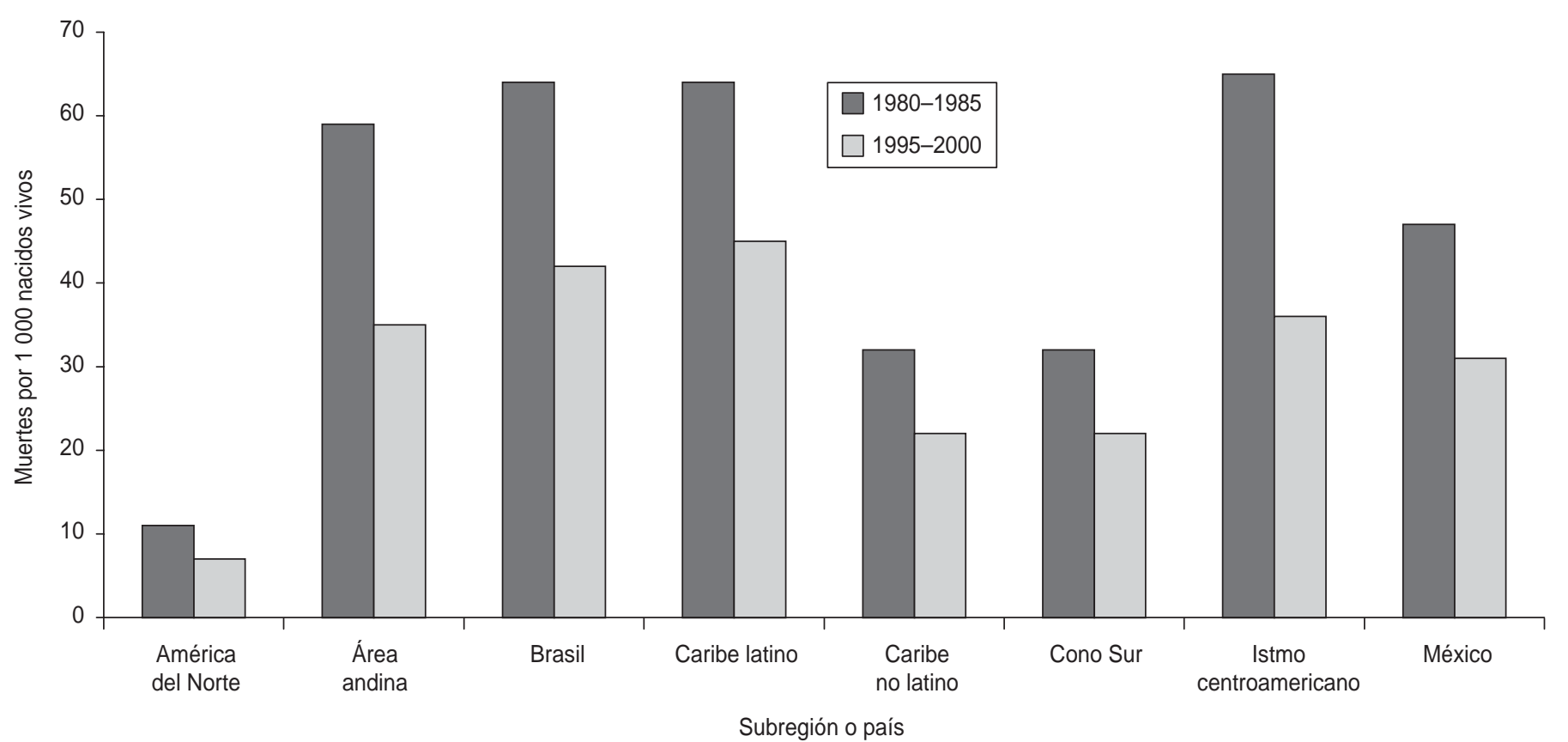

proviene de la Agencia Nacional Aerospacial (NASA) de los Estados Unidos de América (17).

El procesamiento de los datos se realizó mediante el paquete de programas de computación de análisis estadístico SPSS ${ }^{\circledR}$ (18). Se calcularon los valores medianos de la mortalidad infantil, se definieron sus quintiles y se prepararon gráficos de diagramas de caja (boxplots) ${ }^{4}$ para evaluar su distribución, tanto a escala regional como nacional. Para evaluar la relación de la mortalidad con otras variables se utilizaron gráficos de correlación y se calcularon los coeficientes de correlación y regresión. Los datos georreferenciados fueron procesados y analizados en el paquete de programas ArcView GIS $^{\circledR}$ (19). Se prepararon mapas temáticos de coropleta de intervalo para describir la distribución geográfica de las variables, incluidos los cuantiles, como método de clasificación de 10 grupos (deciles), con la superposición de distintas capas

\footnotetext{
5 También se conocen por variables sintéticas en español y por summary measures en inglés.
}

de datos para mostrar la distribución y relación espacial. Se utilizaron las herramientas de superposición y de consulta espacial para calcular los indicadores resumen ${ }^{5}$ de la población.

\section{RESULTADOS}

\section{Análisis regional y subregional}

Los resultados del análisis georreferenciado a una escala de agregación nacional muestran una mejoría en las condiciones generales de vida y salud de las poblaciones en los períodos estudiados. La tasa promedio de mortalidad infantil estimada para toda la Región de las Américas en 1995-2000 fue de 24,8 defunciones por 1000 nacidos vivos, es decir, cerca de 30\% menos que en 1980-1985 (12). Entre las subregiones de las Américas, la situación menos favorable se observó en el Caribe latino, Brasil, el istmo centroamericano y el área andina, y la más favorable en América del Norte, aunque los mayores adelantos entre dichos períodos ocurrieron en el istmo centroamericano (figura 1). Los promedios nacionales de los países variaron entre un mínimo de 5,3 por 1 000 nacidos vivos en Canadá y un máximo de 108,2 en Haití, siendo esta cifra casi 20 veces mayor que la primera, es decir, que sigue habiendo una gran desigualdad. En la figura 2 se muestra la distribución geográfica de la mortalidad infantil, por deciles de riesgo de morir prematuramente, en los países de las Américas. Se observan una tendencia hacia el incremento de la mortalidad y la agregación de tasas más altas hacia la región ecuatorial, en el istmo centroamericano y en algunas islas del Caribe. En cambio, los países con las tasas de mortalidad más bajas son Canadá, Estados Unidos y Cuba en el hemisferio norte, y Chile en el hemisferio sur.

El análisis de la situación de la mortalidad infantil tomando en cuenta el primer nivel de agregación subnacional en 19 países de las Américas para los cuales se dispone de información revela desigualdades importantes en dicho ámbito. La distribución de frecuencias de las tasas de mortalidad para 377 unidades geográficas (estados, departamentos o 
FIGURA 2. Situación de la mortalidad infantil (muertes por 1000 nacidos vivos) en países de las Américas, 1998

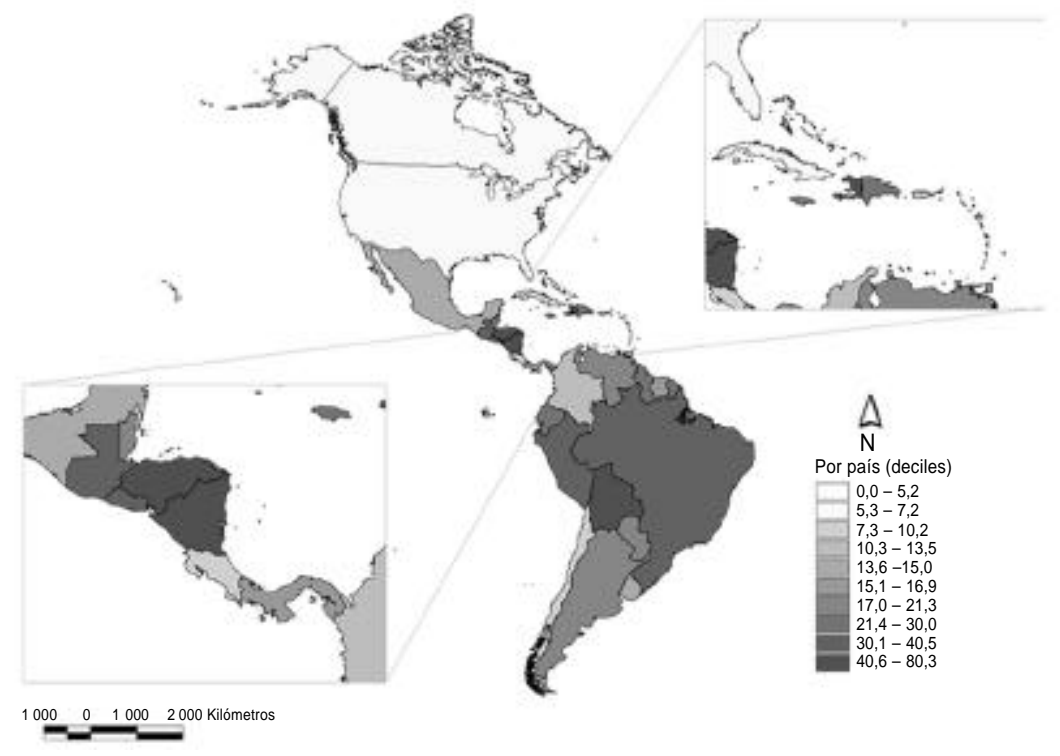

FIGURA 3. Perfil de la mortalidad infantil en algunos países de las Américas. ${ }^{a}$ Distribución de 377 unidades subnacionales, 1995-1999

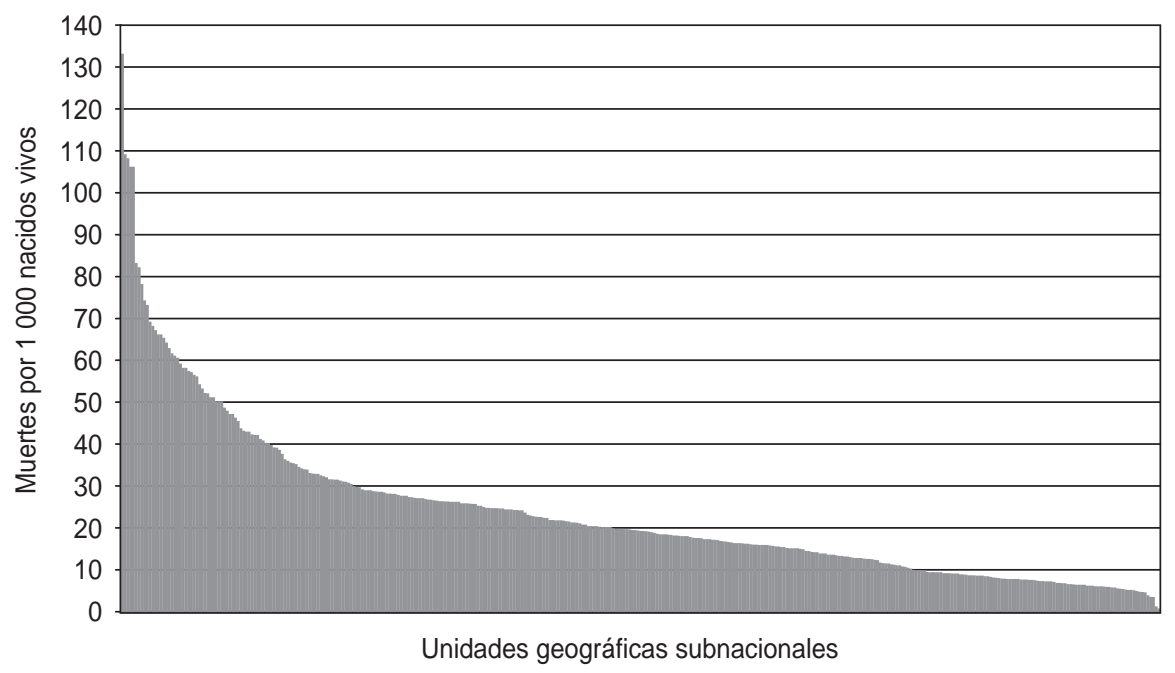

${ }^{a}$ Abarca los siguientes países: Argentina, Belice, Bolivia, Brasil, Canadá, Colombia, Costa Rica, Cuba, Ecuador, Estados Unidos de América, Guatemala, México, Nicaragua, Panamá, Paraguay, Perú, Uruguay y Venezuela.

provincias) de los 19 países mostró una forma marcadamente asimétrica y desigual, con valores mínimos inferiores a 4 muertes por 1000 nacidos vivos y valores máximos superiores a 130 por 1000 , es decir, con diferencias entre cifras extremas de más de 30 veces (figura 3). La mediana de la mortalidad infantil para todas las unidades subnacionales fue de 19,7 defunciones por 1000 nacidos vivos, y los valores medianos mínimo y máximo en el nivel nacional fueron de 5,7 y 83 , respectivamente, lo que representa una diferencia de 15 veces entre valores extremos.

\section{Análisis por países}

La distribución de valores de la mortalidad infantil a escala nacional mostró que también hay grandes desigualdades dentro de algunos de ellos, independientemente del valor mediano que les corresponda (cuadro 1). Por ejemplo, las razones de tasas en Perú, donde la mortalidad infantil es elevada (casi el doble del valor promedio regional), muestra grandes desigualdades internas. Las tasas registradas en las zonas con más alta mortalidad infantil son aproximadamente cuatro veces mayores que las observadas en las de menor mortalidad. Sin embargo, las mayores desigualdades internas que muestra este indicador no se manifiestan necesariamente en los países con las tasas nacionales máximas. De tal manera, en Colombia, donde las tasas de mortalidad son bajas a nivel nacional, la razón de las tasas de mortalidad máximas a las mínimas en los departamentos del país es de 6,2, la mayor entre todos los países analizados. En cambio, en Chile, Uruguay y Cuba, donde las tasas de mortalidad son bajas o mínimas, la razón de las tasas de mortalidad entre las unidades subnacionales también es baja.

La mediana de la mortalidad infantil dentro de los países permite identificar cuatro perfiles de desigualdad en el campo de la salud (figura 4): países con tasas muy bajas (menos de 10 defunciones por 1000 nacidos vivos); países con tasas bajas (cercanas a la media regional de 24 defunciones por 1 000 nacidos vivos); países con tasas altas (de 30 a 40 defunciones por 1000 nacidos vivos), y países con tasas muy altas (40 o más defunciones por 1000 nacidos vivos). También se observan valores muy extremos en la distribución de las tasas de mortalidad infantil en varios países, independientemente del nivel de su mediana, fenómeno que apunta a la presencia de grandes desigualdades. Estos casos extremos se observaron en el departamento de Huancavelica, en Perú, cuya tasa mediana es superior a 40 por 1 
CUADRO 1. Medidas de distribución de la tasa de mortalidad infantil (por 1000 nacidos vivos) en unidades geográficas subnacionales de 19 países de las Américas entre 1995 y 1998

\begin{tabular}{|c|c|c|c|c|c|c|c|}
\hline \multirow[b]{2}{*}{ País } & \multirow[b]{2}{*}{$n^{\mathrm{a}}$} & \multicolumn{6}{|c|}{ Tasa de mortalidad infantil } \\
\hline & & Mínima & Máxima & Razón ${ }^{b}$ & Amplitud & Media & Mediana \\
\hline Argentina & 24 & 9,7 & 34,4 & 3,55 & 24,7 & 20,8 & 20,05 \\
\hline Bolivia & 9 & 50,0 & 133,0 & 2,66 & 83,0 & 87,3 & 83,0 \\
\hline Brasil & 27 & 19,66 & 74,07 & 3,77 & 54,41 & 40,0 & 35,02 \\
\hline Canadá & 11 & 4,6 & 12,2 & 2,65 & 7,6 & 6,43 & 5,7 \\
\hline Chile & 13 & 8,9 & 14,9 & 1,67 & 6,0 & 11,39 & 11,30 \\
\hline Cuba & 15 & 5,4 & 10,3 & 1,91 & 4,9 & 8,09 & 8,0 \\
\hline Ecuador & 20 & 10,9 & 32,7 & 3,00 & 21,8 & 19,9 & 18,45 \\
\hline Estados Unidos de América & 51 & 4,4 & 14,9 & 3,39 & 10,5 & 7,42 & 7,4 \\
\hline Guatemala & 22 & 24,02 & 58,03 & 2,42 & 34,01 & 38,1 & 35,52 \\
\hline México & 33 & 14,0 & 42,8 & 3,06 & 28,8 & 24,0 & 22,2 \\
\hline Nicaragua & 17 & 12,63 & 40,12 & 3,18 & 27,49 & 23,8 & 22,4 \\
\hline Panamá & 10 & 11,1 & 29,8 & 2,68 & 18,7 & 19,2 & 18,1 \\
\hline
\end{tabular}

Fuente: Referencia 22

a La $n$ representa el número de unidades subnacionales contempladas.

${ }^{\mathrm{b}}$ Representa la razón de la tasa máxima a la mínima.

FIGURA 4. Mortalidad infantil en algunos países de las Américas, 1995-1999. Distribución de 377 unidades geográficas municipales

el espacio geográfico-poblacional de los

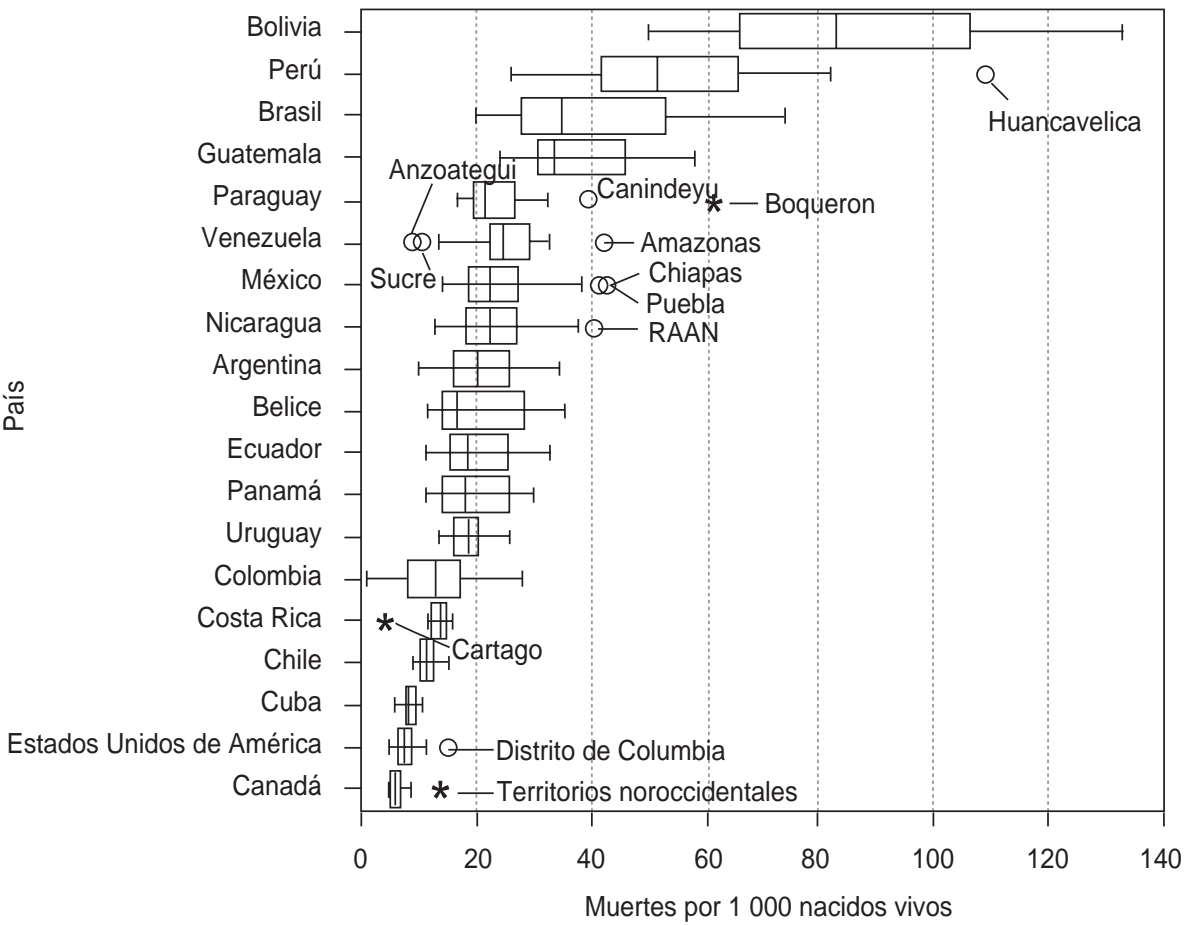

000 nacidos vivos, y en el Distrito de Columbia, en los Estados Unidos, cuyo valor mediano es menor de 10 por 1000 .
En ambos casos, la razón de la tasa más alta a la más baja es mayor de 3:1.

El despliegue de valores de las tasas de mortalidad infantil subnacionales en países seleccionados permite identificar con mayor precisión la magnitud de las desigualdades y su agregación o dispersión. Geográficamente, se observó una distribución de mosaico que reproduce las desigualdades encontradas a escala regional, incluidas importantes diferencias entre países, sin importar el valor mediano nacional (figura 5). Por ejemplo, en Canadá y en Estados Unidos, que tienen las tasas medianas más bajas de todos los países americanos, se distinguen en el norte y en el sureste, respectivamente, áreas con mayor mortalidad que contrastan con el resto de cada país. Si se hace un acercamiento en el mapa hacia el área andina y Brasil (figura 6), se pueden distinguir con mayor claridad las diferencias entre unidades subnacionales y entre países. Ahí también se observan grupos de unidades geográficas con las mayores tasas de mortalidad en las zonas más cercanas a las áreas tropicales ecuatoriales de los estados amazónicos y en los extremos oriental y occidental de Brasil, incluida la mayor parte de Perú y Bolivia. Debe tenerse en cuenta para la interpretación de los resultados que la comparación 
FIGURA 5. Mortalidad infantil (por 1000 nacidos vivos) en países de las Américas, por unidades geográficas subnacionales de primer nivel, entre 1995-2000

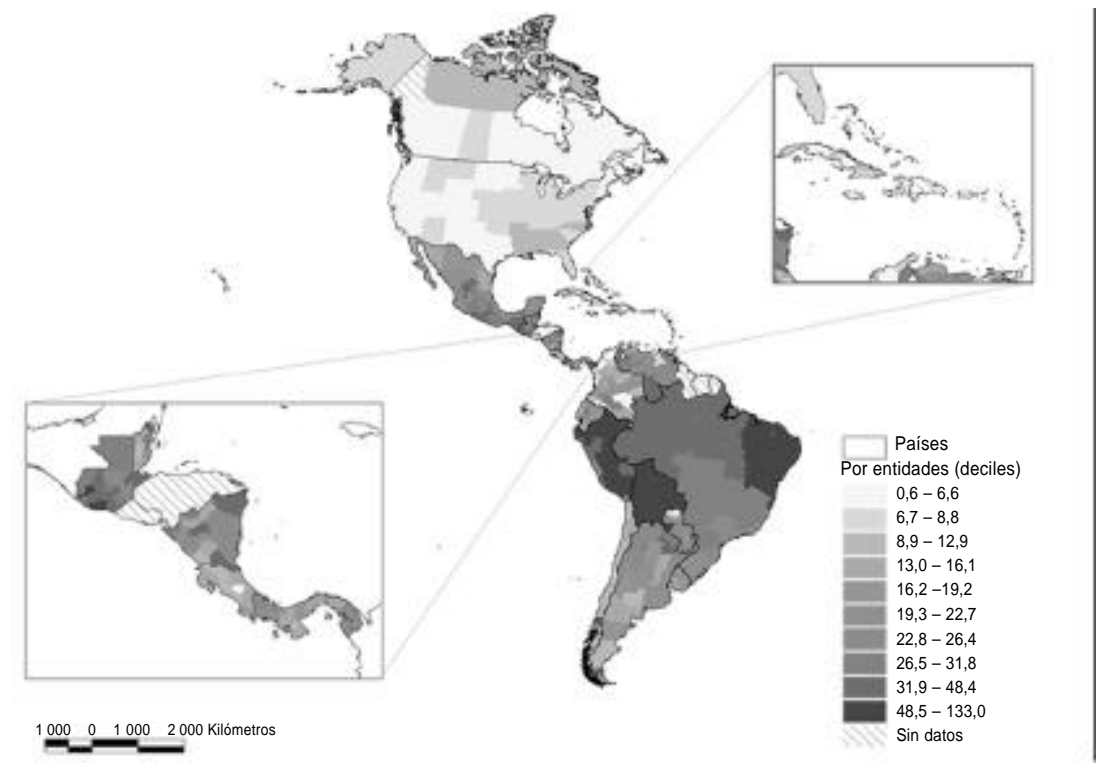

FIGURA 6. Mortalidad infantil (por 1000 nacidos vivos) en países de las Américas, por unidades geográficas subnacionales de primer nivel, entre 1995-2000. Vista del sur de la Región

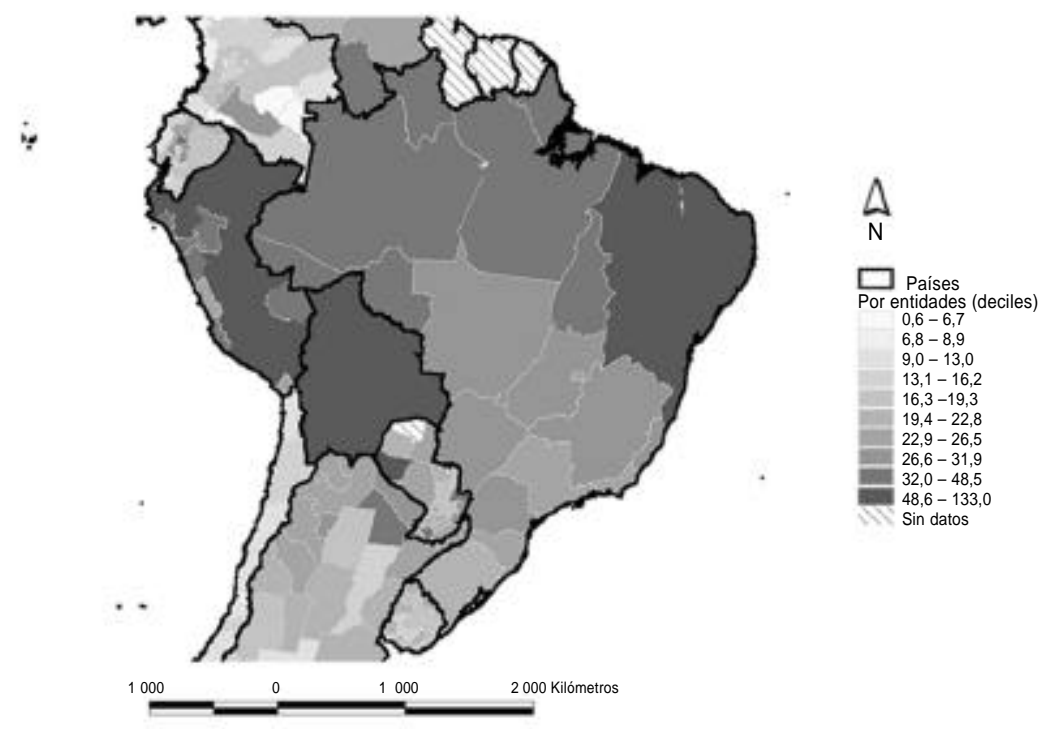

anterior abarca la distribución agrupada de todos los países, procedimiento que podría ser inadecuado, dadas las importantes diferencias en la disponibilidad de recursos de salud y en la forma de distribuirlos. Debido a ello, visión de la distribución de la mortalidad infantil y de algunos de sus factores determinantes más pertinente para tomar decisiones a escala local. Se observó que existen grandes desigualdades en la distribución de las tasas de mortalidad infantil entre los 5507 municipios del país; $10 \%$ de las unidades geográficas tiene más de 70 muertes por 1000 nacidos vivos —el doble del valor mediano nacional - y $10 \%$ menos de 18 por 1000 (figura 7). La razón de las tasas de mortalidad máxima y mínima indica que al $10 \%$ superior le corresponde un riesgo cerca de cuatro veces mayor que al inferior. Al examinarse los valores pertenecientes a municipios individuales, la razón entre la situación menos favorable $(99,4$ por 1000 nacidos vivos) y la más favorable (3,4 por 1000$)$ indica un riesgo 30 veces mayor en el primer caso. Esta razón de tasas es similar a la encontrada entre estados, aunque la curva de distribución es menos asimétrica que la observada para algunos países de la Región. En los estados de Brasil, la distribución de las tasas de mortalidad por municipios muestra grandes desigualdades entre estados y dentro de cada estado (figura 8). Se destacan algunas unidades con valores cercanos a cero en los estados con medianas próximas a 80 muertes por 1000 nacidos vivos, debido posiblemente a coberturas locales o estimaciones inadecuadas, mientras que las unidades que tienen valores cercanos a cero en los estados con la mortalidad más baja (cerca de 20 por 1000 ) pueden ser un genuino reflejo de los niveles muy bajos propios de zonas urbanas más desarrolladas.

La distribución geográfica de la mortalidad infantil a escala municipal en Brasil muestra un patrón de desigualdades entre grupos de municipios con distintos riesgos que tiene forma de parches irregulares. Ese patrón concentra las tasas más altas en los estados del noreste y en la cuenca alta del Amazonas en el extremo occidental del país principalmente, donde los valores ascienden a más de 60 muertes infantiles por 1000 nacidos vivos (figura 9). En cambio, en los municipios de los estados del sur, las tasas de mortalidad son, por lo general, menores de 25 por 1 
FIGURA 7. Perfil de la mortalidad infantil por municipios de Brasil. Distribución correspondiente a 5507 unidades geográficas, 1998

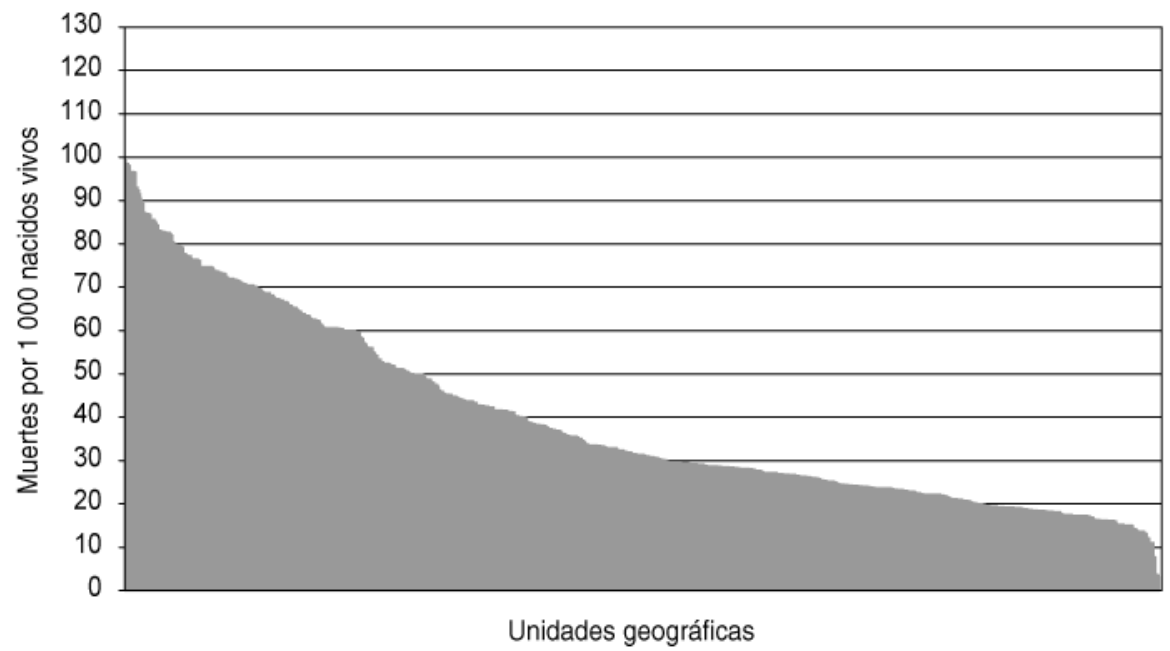

FIGURA 8. Mortalidad infantil por estados de Brasil. Distribución correspondiente a 5507 unidades geográficas municipales, 1998

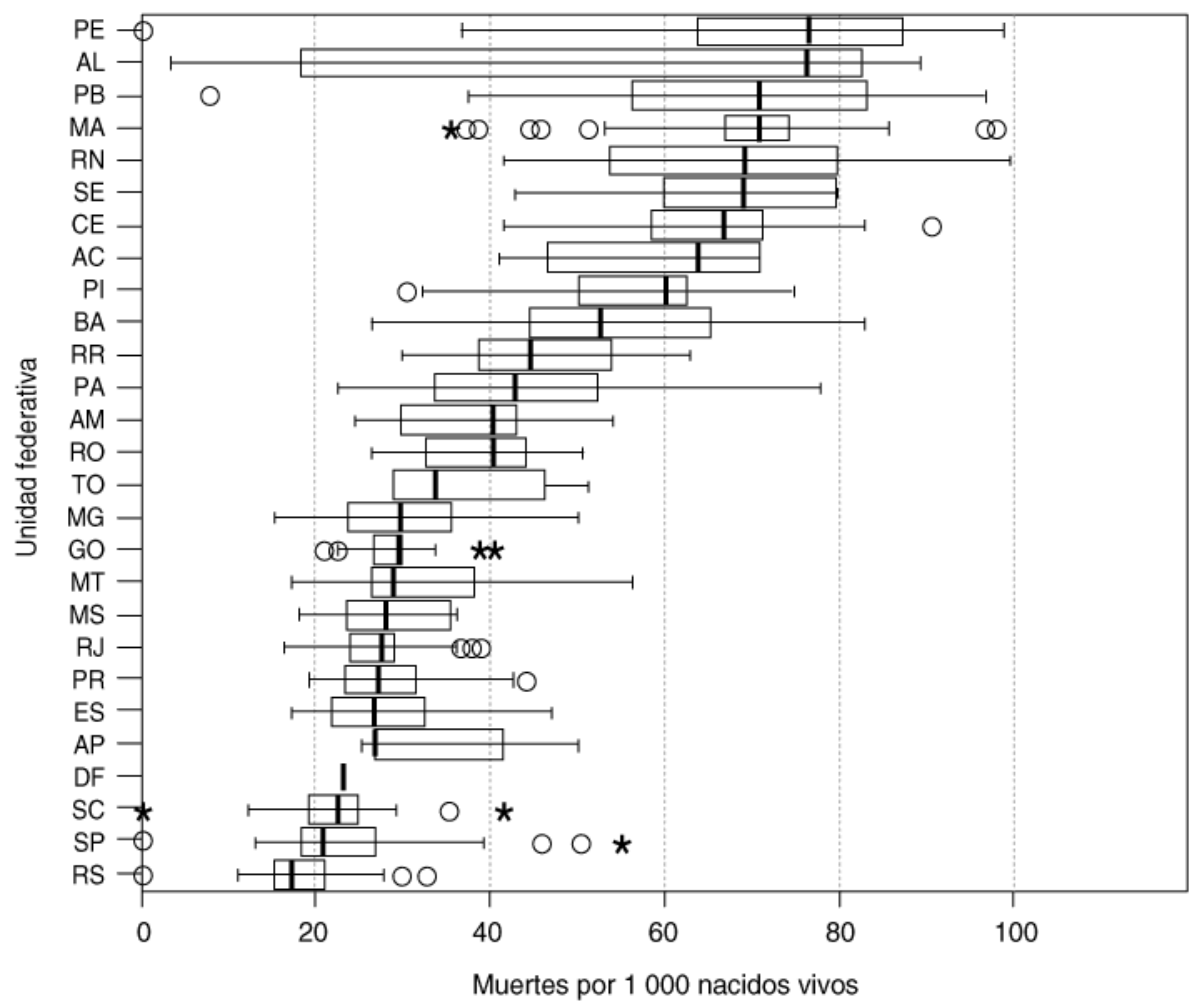

000. El analfabetismo femenino se incluyó en estos análisis por ser uno de los factores determinantes importantes de la mortalidad infantil y por ser un indicador que refleja el nivel observada en el caso de la mortalidad (figura 10). En este mapa se agregó una base cartográfica con las áreas de reservas indígenas de Brasil, a fin de determinar si estas coincidían con las áreas de mayor prevalencia de analfabetismo femenino, fenómeno que se observó en particular en el noroeste. Aunque la mortalidad infantil y el analfabetismo femenino guardan, en general, una fuerte correlación directa $(r$ $=0,71$ ) (figura 11), visualmente es difícil definir esta relación en los mapas, aunque ambas variables ocupan espacios geográficos bastante similares. Por lo tanto, para identificar las áreas donde hay mayor mortalidad infantil y analfabetismo femenino se sobrepusieron ambos indicadores con la imagen de satélite en un nuevo mapa, con el fin de mostrar la relación con otros elementos geográficos, como la cuenca del Amazonas en el norte (figura 12). El uso de distintos tonos entre blanco y negro permite distinguir las áreas donde los valores de ambos indicadores coinciden en su expresión más alta, principalmente en el extremo oriental de la cuenca del Amazonas.

Ecuador. La mortalidad infantil en los 214 cantones de Ecuador mostró un perfil de distribución de valores menos heterogéneo que en Brasil. Ese perfil está indicado por la pendiente de la curva de distribución de dicho indicador (figura 13). Asimismo, las tasas de mortalidad en los cantones son más altas que en Brasil, si se considera que $10 \%$ de las unidades geográficas tienen valores mayores de 82 por 1000 nacidos vivos y, en el otro extremo, un $10 \%$ adicional tiene valores menores de 35 por 1000 nacidos vivos. La razón del valor máximo al mínimo, que es de 2,3:1, representa, sin embargo, una importante desigualdad en el riesgo de mortalidad infantil, aunque es alrededor de la mitad de lo observado en los municipios de Brasil. En las provincias del Ecuador, la distribución de la mortalidad infantil por cantones mostró menos heterogeneidad y menores desigualdades que en los estados brasileños (figura 14).

En Ecuador, la mortalidad infantil y el analfabetismo femenino muestran desigualdades similares a las de Brasil 
FIGURA 9. Mortalidad infantil (por 1000 nacidos vivos) en Brasil, por unidades geográficas subnacionales de segundo nivel, 1998
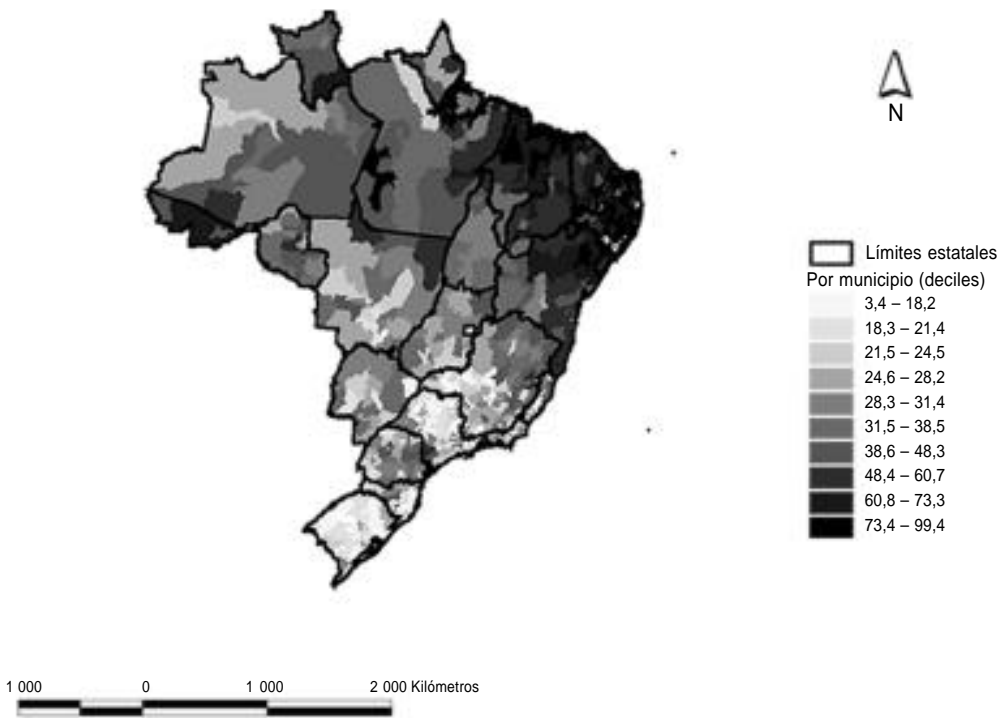

FIGURA 10. Analfabetismo femenino (\%) en Brasil, por unidades geográficas subnacionales de segundo nivel en 1998, y áreas de reserva indígena
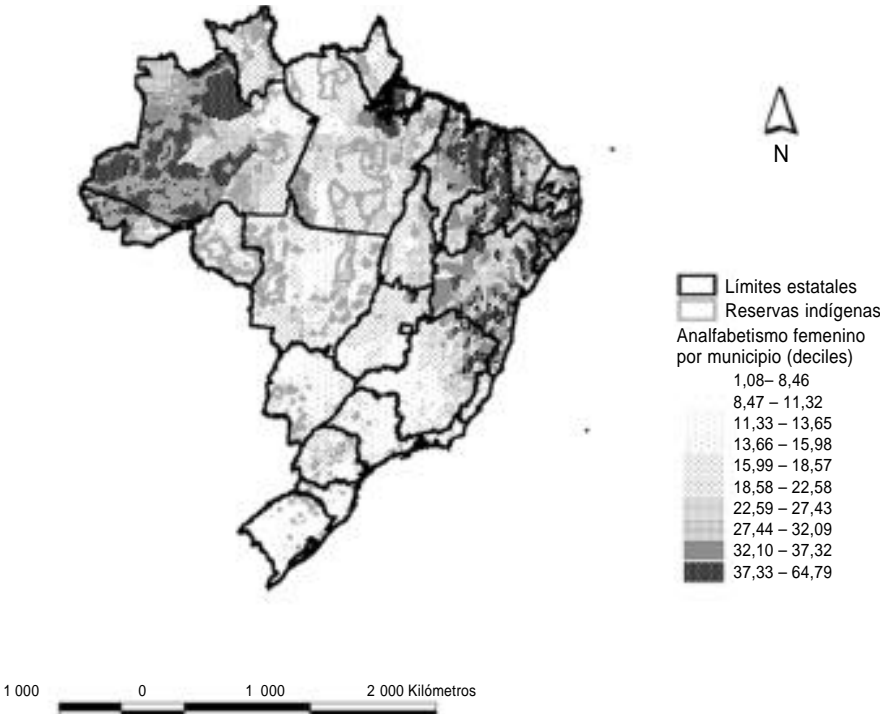

cuando se examinan por zonas geográficas. En el caso de la mortalidad infantil se encontraron algunos conglomerados (clusters) de cantones con tasas cercanas a 64 por 1000 nacidos vivos, principalmente en el centro del país, en las regiones del este amazónico y en la frontera norte (figura 15). analfabetismo femenino. Se observó, no obstante, una marcada correlación directa $(r=0,76)$ entre la mortalidad infantil y el analfabetismo (figura 17). Este hecho corrobora y respalda los resultados que apuntan hacia una asociación entre estos dos indicadores en Brasil, lo cual indica que cualquiera de los dos se puede usar para monitorear las desigualdades de la mortalidad infantil.

Además del analfabetismo femenino, en el análisis de las desigualdades de la mortalidad infantil en Ecuador se incluyó también la desnutrición crónica (definida como un déficit en la talla para la edad) en niños menores de 5 años. Este indicador puede considerarse tanto un factor determinante de la mortalidad infantil como un efecto que la situación socioeconómica ejerce sobre la salud. Las tasas de prevalencia de desnutrición en los cantones mostraron un patrón de distribución con un conglomerado de mayor riesgo en la parte central del país, donde los valores ascienden a más de $54 \%$ (figura 18). En esa distribución geográfica se destacan dos aspectos. Por un lado, hay un patrón lineal indicativo de una relación con algún factor de carácter ambiental. Por otro, sobre dicha línea se observan algunos cantones de menor riesgo, los cuales coinciden hacia el norte con las provincias de la capital del país o cercanas a ella, y hacia el sur con las provincias de Guayas y El Oro, que también figuran entre las que poseen más recursos de Ecuador.

Para mostrar la capacidad para manejar múltiples variables con un sistema de información geográfica (SIG) se preparó un mapa con el despliegue conjunto de los indicadores de mortalidad infantil, analfabetismo femenino y desnutrición, y en el mapa se observa un patrón de desigualdad en el centro del país. En un intento por explicar este patrón, se incluyó el relieve continental, con la superposición de los valores de los indicadores en el mapa (figura 19). Resulta evidente que la mayoría de los cantones de mayor riesgo coinciden y se alinean a lo largo de la cordillera de los Andes. En estos cantones andinos también se advierten altos niveles de pobreza en poblaciones indígenas y diversas condiciones de 
FIGURA 11. Correlación entre mortalidad infantil y analfabetismo femenino en municipios de Brasil, 1998

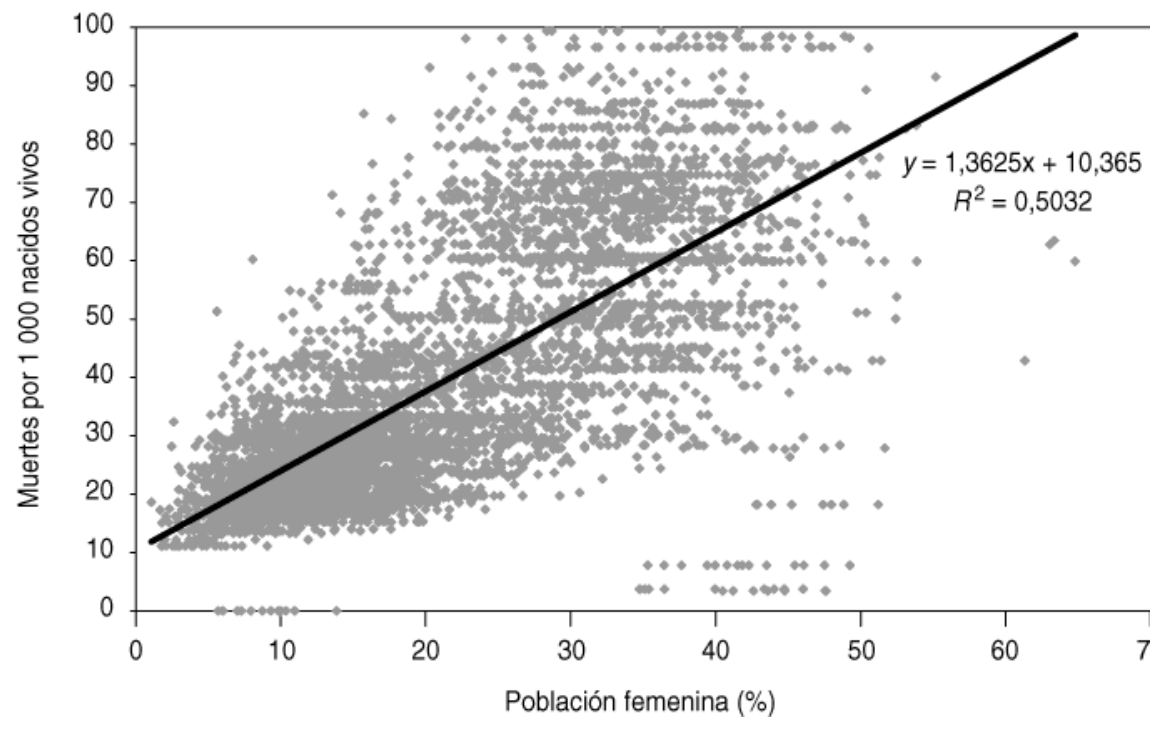

FIGURA 12. Mortalidad infantil (por 1000 nacidos vivos) y analfabetismo femenino (\%) en Brasil, por unidades geográficas subnacionales de segundo nivel en 1998, sobre el relieve continental

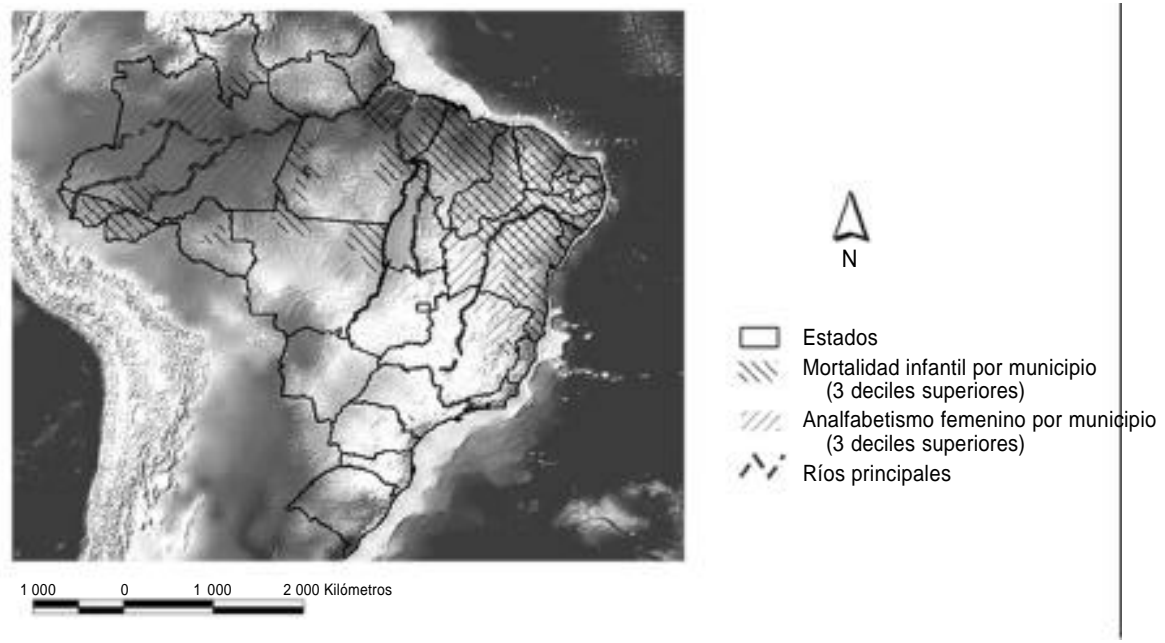

erosión del suelo que limitan la producción y la disponibilidad de alimentos. Sin embargo, hay otros grupos de cantones de alto riesgo en las zonas costeras fronterizas del norte y en la cuenca alta del Amazonas, en el este del país, donde hay otros factores condicionantes más importantes, como un acceso limitado a servicios, entre ellos otro deberán adecuarse a la importancia de esos factores.

Finalmente, debido a que los recursos de salud suelen ser limitados y es preciso usarlos cada vez más con mayor eficiencia, mediante consultas lógicas estructuradas del SIG se seleccionaron las unidades geográficas críticas, es decir, aquellas donde coincidieron simultáneamente los niveles de riesgo más altos (por ejemplo, los tres deciles superiores de cada indicador). Los criterios o valores de corte utilizados fueron una tasa mínima de mortalidad infantil de 63,6 defunciones por 1000 nacidos vivos; una prevalencia mínima de analfabetismo femenino de $21,5 \%$, y una prevalencia mínima de desnutrición de $54,3 \%$. Este proceso permitió identificar un subconjunto de 33 unidades geográficas en el mapa que deberían ser objeto prioritario de las intervenciones de salud (figura 20). Gracias a otras herramientas de consulta espacial de datos que los SIG poseen, se pudo determinar que la mediana de la tasa de mortalidad infantil del conjunto seleccionado era de 83 por 1000 nacidos vivos, mientras que la del resto del país era de 56 por 1 000. Con estas herramientas de consulta se puede calcular la población de cada uno de estos estratos de riesgo $\mathrm{y}$, por tanto, estimar los recursos que se requieren para satisfacer las necesidades de salud de cerca de un millón de personas, es decir, $16 \%$ de la población total del país a principios de 1990.

\section{DISCUSIÓN}

Durante las dos últimas décadas, la situación de salud de los países de las Américas ha mejorado sustancialmente, a juzgar por los valores promedio de los indicadores nacionales. No obstante, diversos países y grupos de población continúan rezagados, como ponen de manifiesto las cifras promedio de sus indicadores de salud, nivel de vida y acceso a servicios de salud. Estos promedios nacionales han servido fundamentalmente para orientar políticas generales, ya que retratan las desigualdades a escala nacional, y para generar políticas y metas regionales (como la de salud para todos en 
FIGURA 13. Perfil de mortalidad infantil en Ecuador por cantones. Distribución de 214 unidades geográficas, 1995

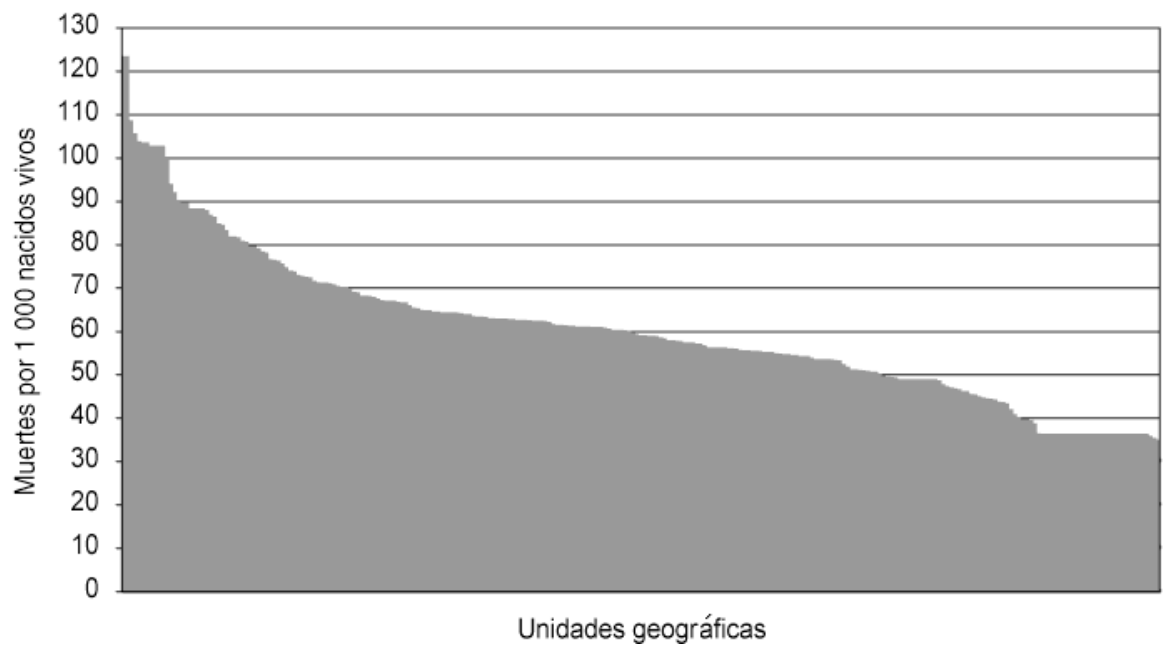

FIGURA 14. Mortalidad infantil (por 1000 nacidos vivos) por provincias de Ecuador, 1995. Distribución de 214 unidades geográficas cantonales

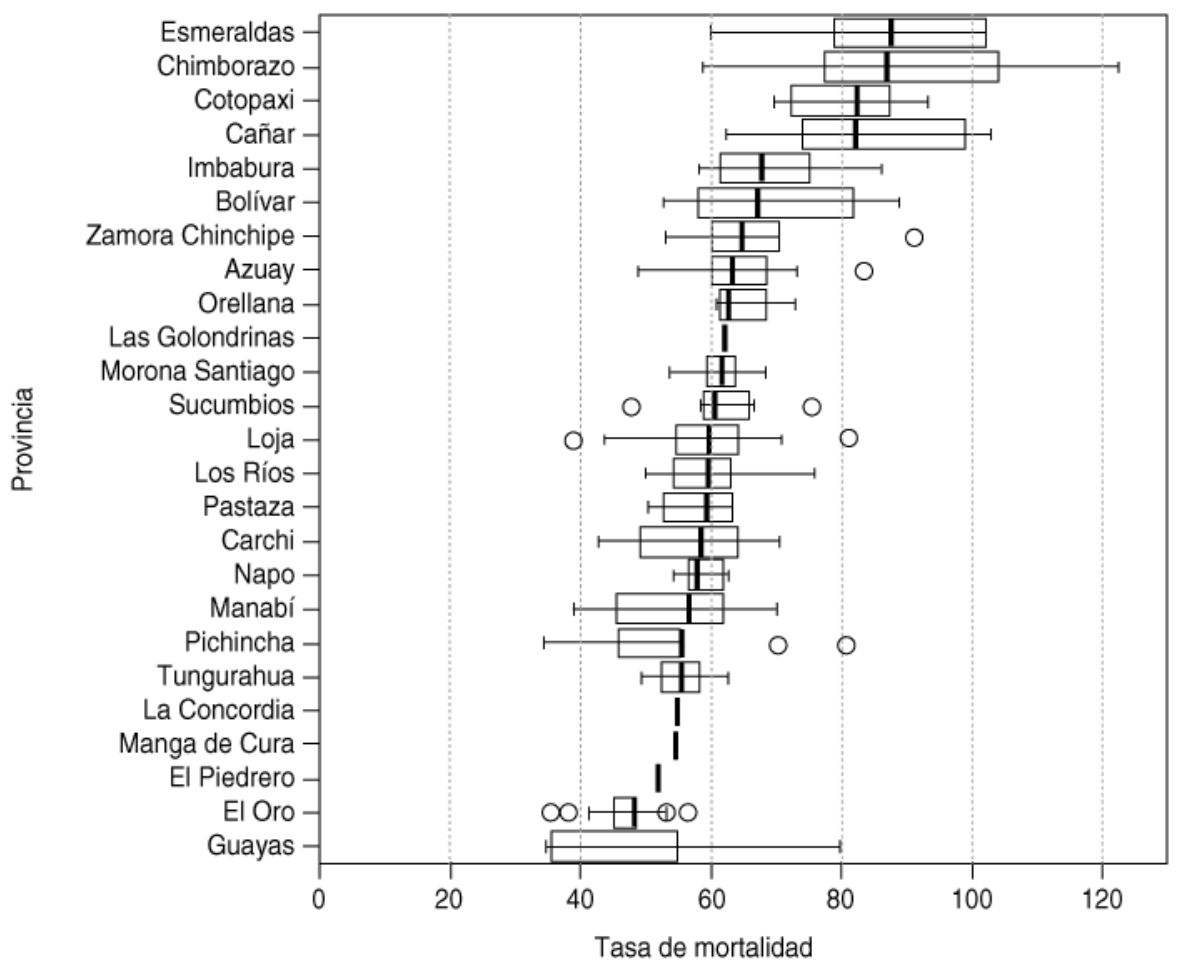

el año 2000). Sin embargo, este enfoque no ha permitido formular adecuadamente, a escala local, propuestas específicas para hacer intervenciones sanitarias en los países que faciliten el uso más eficiente $y$ equitativo de los recursos. Estudios promedio de los indicadores de grandes áreas (21), reforzando la necesidad de realizar análisis en áreas más pequeñas y homogéneas en su perfil socioepidemiológico que sean pertinentes para las decisiones a escala local.

Para que los responsables de las políticas y programas sociales y de salud puedan responder con medidas más eficaces de prevención y control de daños y de promoción de la salud, hay que mejorar algunos aspectos de los análisis de la situación de salud a fin de medir las desigualdades existentes. Entre ellos figuran los siguientes:

1) Simplificar y sintetizar la información básica, de manera que les sea accesible a las autoridades decisorias. Los informes voluminosos que suelen prepararse para analizar la situación de salud desalientan al lector.

2) Definir los patrones de distribución de salud que, por un lado, apunten hacia factores determinantes relacionados con el espacio geográfico y, por otro, faciliten la identifica ción de estratos epidemiológicos de espacio-población con diferentes factores de riesgo determinantes. Hasta ahora no hay posibilidades de hacer un análi-sis de esta naturaleza sin la ayuda de una representación geográfica en un mapa.

3) Definir la proximidad o vecindad entre elementos o unidades de análisis. Es muy difícil determinar esta proximidad a partir de datos organizados en un cuadro o en una base de datos y focalizar las intervenciones local o regionalmente.

4) Determinar las relaciones espaciales de variables e indicadores de riesgo que coinciden en el mismo lugar y población.

5) Realizar consultas de datos basadas en variables geográficas. Las capacidades de los SIG facilitan este proceso.

Los SIG son instrumentos esenciales para medir desigualdades de salud e identificar grupos y áreas con mayores problemas a escala local. En el campo de la salud, los SIG se han descrito como un conjunto de datos de orden espacial y sanitario que interactúan, permitiendo analizar y sintetizar una gran cantidad de datos con el fin de describir una situación de salud, efectuar análisis epidemiológicos y orientar y evaluar la gestión, las 
FIGURA 15. Mortalidad infantil (por 1000 nacidos vivos) en Ecuador, por unidades geográficas subnacionales de segundo nivel, en 1995

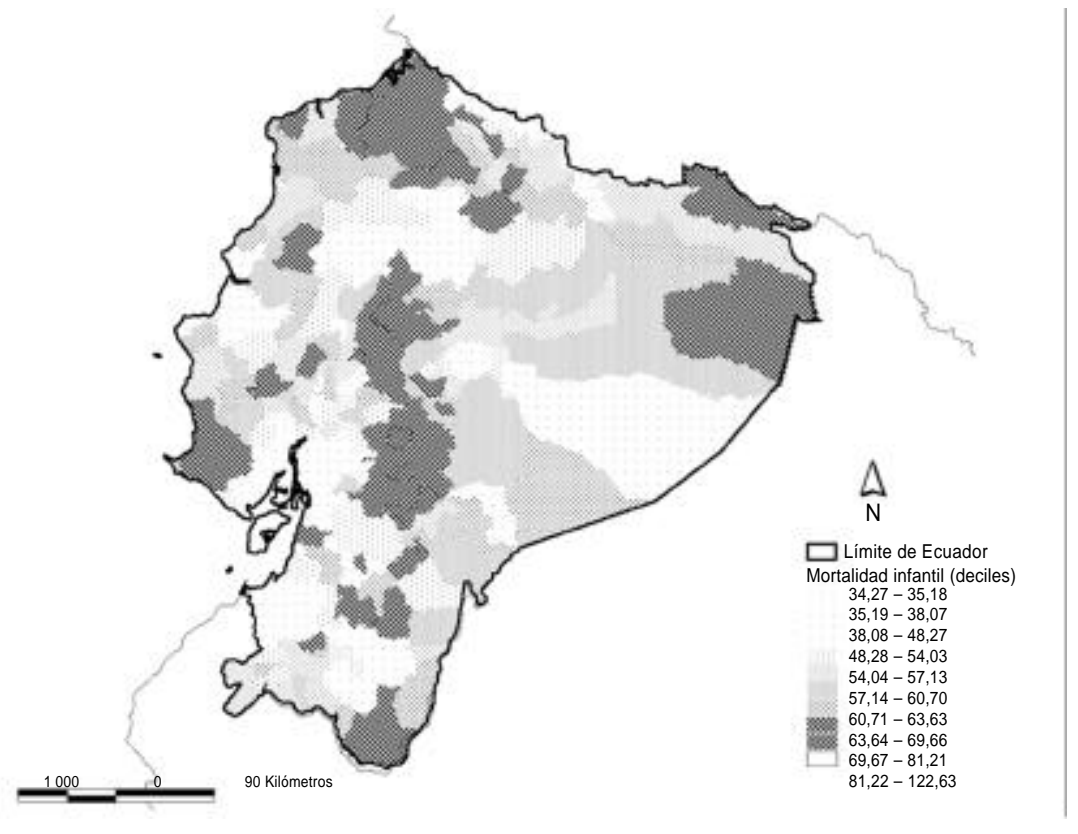

FIGURA 16. Prevalencia de analfabetismo femenino (\%) en Ecuador, por unidades geográficas subnacionales de segundo nivel, en 1995

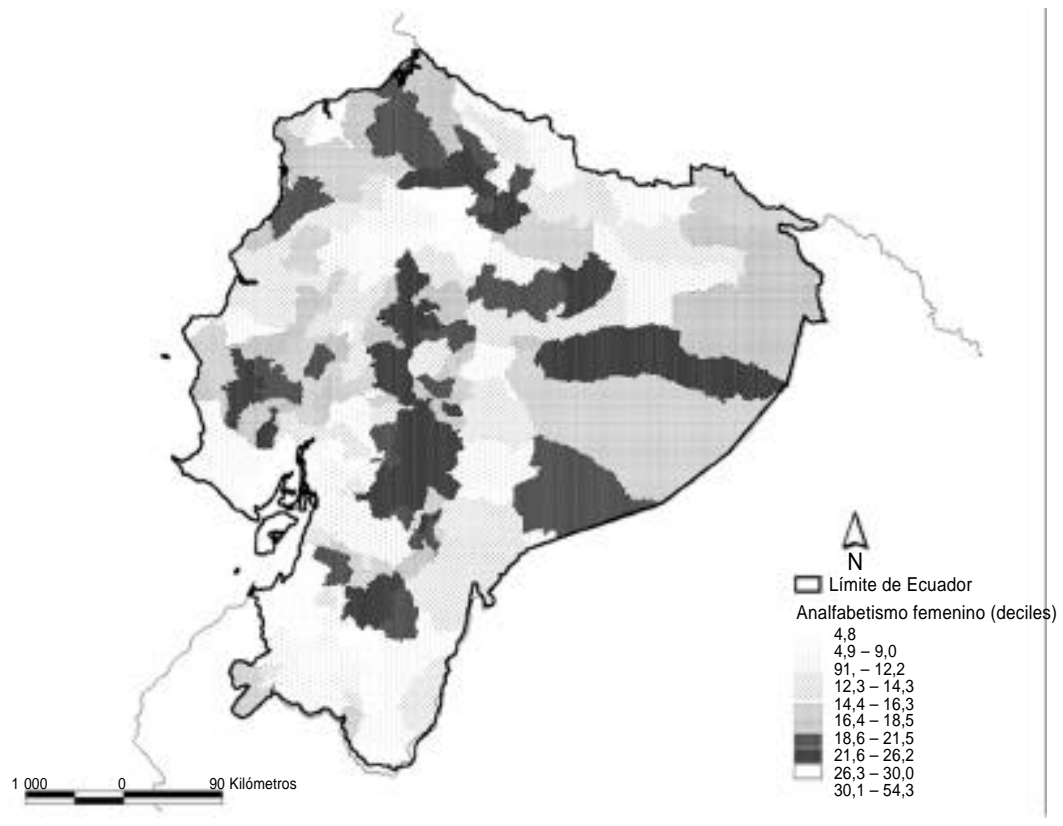

intervenciones y la toma de decisiones en el ámbito de la salud (22). Una de las ventajas adicionales específicas de estos sistemas, además de su capacidad para integrar análisis exploratorios, tales como los gráficos de distribución de frecuencias y los diagramas de caja, junto con la generación de mapas temáticos -incluida la superposición de diversas capas de información de distintos tipos de datos epidemiológicos y sociales, y las consultas espaciales de los SIG permiten identificar, medir y monitorear las desigualdades de salud e identificar los grupos de población y las áreas con mayores necesidades de salud insatisfechas.

En el presente trabajo se presentan los resultados del monitoreo de las desigualdades de la mortalidad infantil en el ámbito subnacional de algunos países de las Américas, habiéndose identificado así diversos estratos epidemiológicos conformados por conglomerados de unidades geográfico-poblacionales con niveles de riesgo y factores determinantes similares. Estos hallazgos coinciden con los de estudios realizados en otros países y regiones. Por ejemplo, en México se encontraron a escala estatal diferencias regionales en los patrones de mortalidad general que estaban asociados, a su vez, con la interacción de niveles socioeconómicos y con la prestación de servicios de salud (23). El haber encontrado conglomerados de unidades geográficas municipales con un mayor riesgo epidemiológico, los cuales representan la fracción más importante del problema de salud pero abarcan, a su vez, una pequeña fracción de la población, tiene implicaciones importantes para formular políticas dirigidas a grupos vulnerables. Al focalizar las intervenciones de salud más eficaces en dichas áreas se resolverá la mayor parte del problema. La importancia de este aspecto ha sido demostrada previamente en el caso de la malaria en Brasil (22), donde en 199330 municipios -que representaban menos de $1 \%$ de la población total- producían más de $60 \%$ de los casos.

Para identificar las áreas críticas cuando se contemplan diferentes indicadores no solo es importante determinar las brechas de salud entre un área y otra, sino tener también presentes la fracción y el perfil de la población que vive en cada estrato. Estos elementos combinados dan como indicación la fracción del riesgo o el riesgo atribuible poblacional que puede eliminarse mediante cada intervención 
FIGURA 17. Correlación entre mortalidad infantil (MI) y analfabetismo femenino (AF) en Ecuador, por unidades geográficas subnacionales de segundo nivel, en 1995

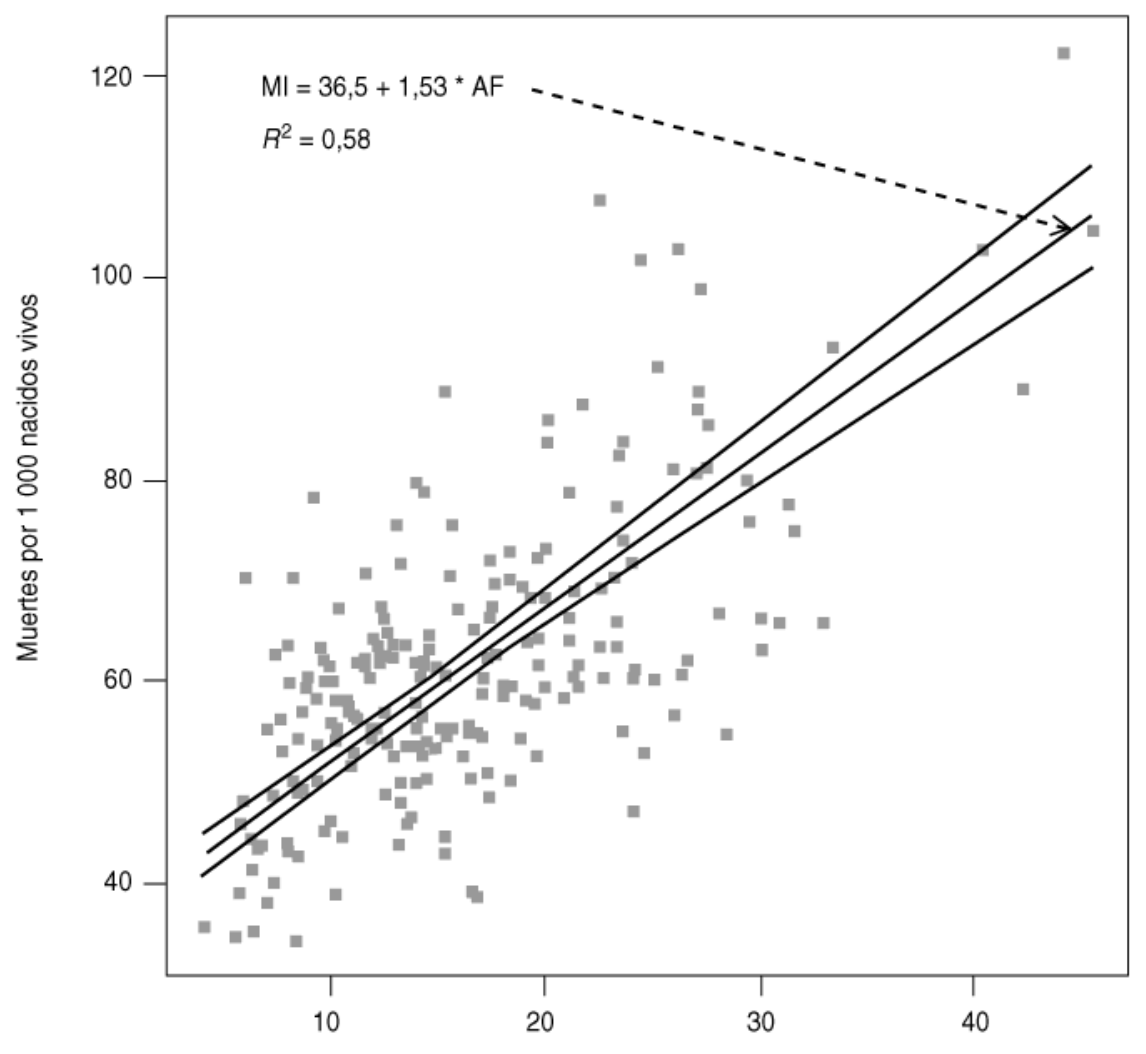

Prevalencia de analfabetismo (\%)

FIGURA 18. Prevalencia de desnutrición en menores de 5 años (\%) en Ecuador, por unidades geográficas subnacionales de segundo nivel, en 1995

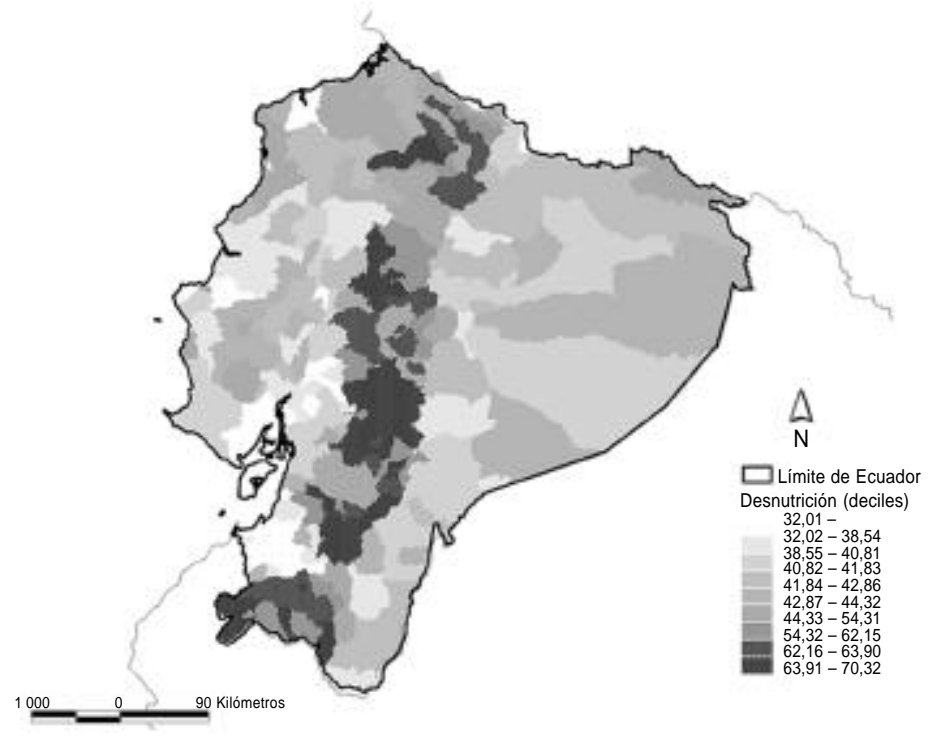

sanitaria específica, por lo que tienen gran importancia en salud pública.

$\mathrm{Al}$ interpretar los resultados de análisis de la situación de salud como estos, algunos aspectos deberán tenerse muy presentes. Por un lado, la agregación de datos en grupos o unidades de análisis compuestas de agregados de personas conlleva la posibilidad de incurrir en la denominada "falacia ecológica" (24), es decir, de dar por sentado que las observaciones efectuadas en unidades geográficas representan el riesgo de cada persona individualmente. Sin embargo, la plausibilidad de que así sea, proveniente de estudios etiológicos, y la coincidencia entre los resultados de distintos estudios observacionales a nivel individual $y$ colectivo, respaldan la validez de las observaciones. En este sentido, la elaboración de mapas de áreas pequeñas ha sido útil para identificar conglomerados regionales y locales y para generar hipótesis etiológicas, como se desprende de los análisis del atlas de mortalidad de los Estados Unidos, donde se observa una asociación geográfica entre diferentes patrones de distribución de la mortalidad por diversos tipos de causas, por un lado, y factores que definen los estilos de vida, así como los niveles de pobreza $y$ urbanización, por el otro (25).

Cabe subrayar que el propósito de este tipo de estudios es proporcionar a las autoridades de salud elementos sintéticos que sirvan de base para sus decisiones. Por otro lado, los datos obtenidos de rutina en los registros habituales de los países revisten gran importancia, como también los utilizados en estos análisis de situación o los que provienen de otras fuentes, incluidas las encuestas. Si bien estas fuentes son complementarias y una no descarta a la otra, como se da a entender en ocasiones, los datos recogidos por encuesta se obtienen por muestreos de viviendas o individuos, y su representatividad podría verse limitada a determinadas regiones debido al tamaño muestral. Esta circunstancia limitaría la capacidad de identificar conglomerados (clusters) y desigualdades en niveles más desagregados, como los municipios (nivel local), y por tanto serían de menor utilidad para planear intervenciones locales, actividad que es de interés fundamental 
FIGURA 19. Mortalidad infantil (por 1000 nacidos vivos), analfabetismo femenino (\%) y desnutrición en menores de 5 años (\%) en Ecuador, por unidades geográficas subnacionales de segundo nivel en 1995, sobre el relieve continental

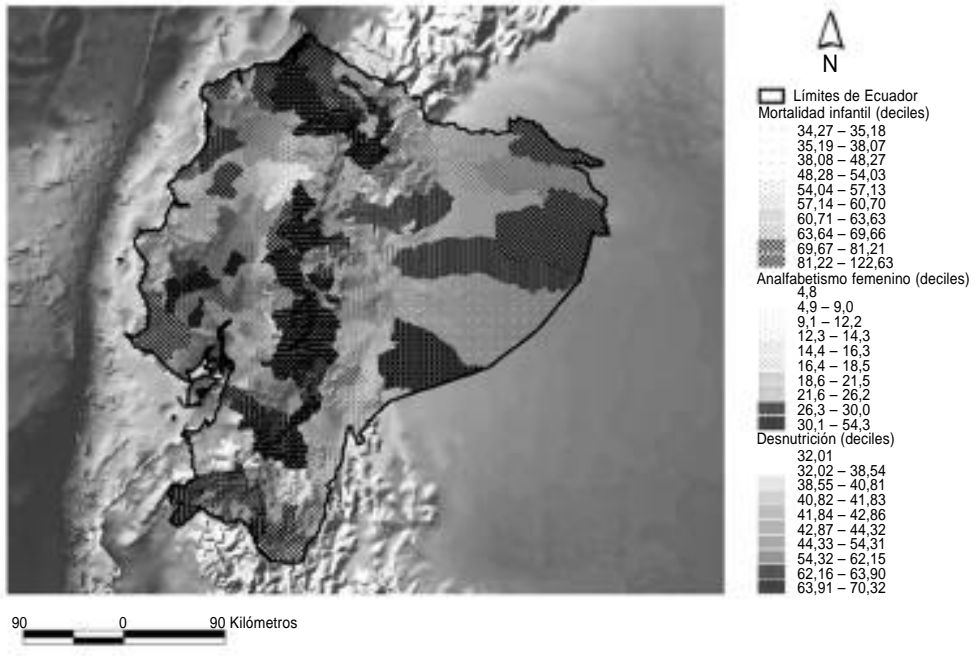

FIGURA 20. Áreas críticas de mortalidad infantil (por 1000 nacidos vivos), analfabetismo femenino (\%) y desnutrición en menores de 5 años (\%) en Ecuador, por unidades geográficas subnacionales de segundo nivel en 1995, sobre el relieve continental
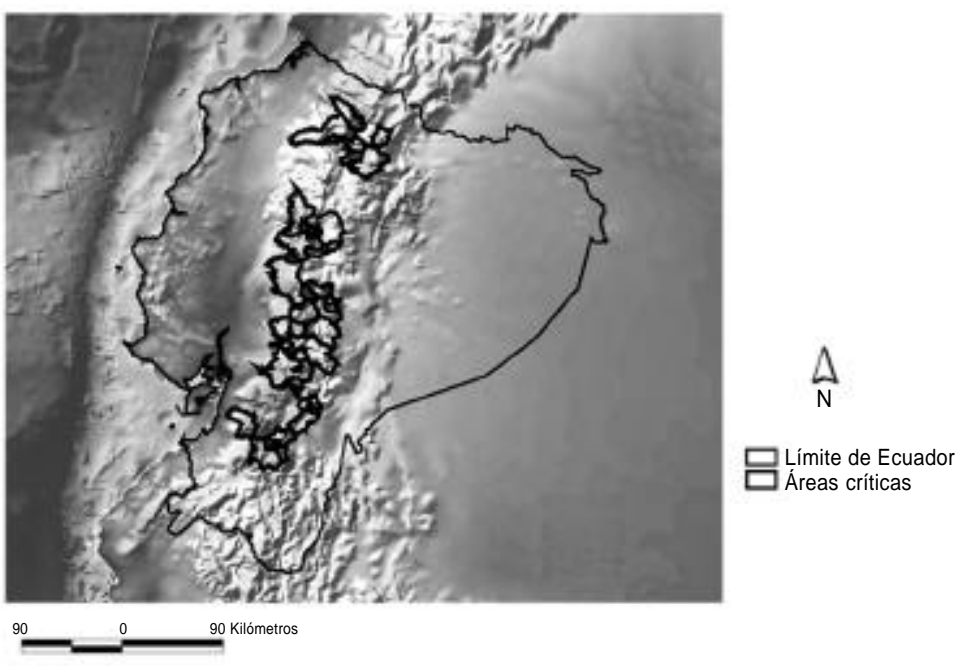

para las instancias decisorias en este período de descentralización de los servicios de salud.

En regiones del norte de Brasil se reconocen algunas limitaciones en la calidad de los datos de mortalidad infantil y analfabetismo que podrían llevar a interpretaciones sesgadas. Se sabe que el embargo, el efecto que tendrían estas últimas en la interpretación de los resultados observados sería también limitado. Dado que la fracción de los municipios con un nivel de analfabetismo inesperado es menor de 1\%, aun si dichos municipios fuesen eliminados no se modificarían sustancialmente los resultados. Por otro lado, la existencia de otras situaciones y países con datos similares apoya la uniformidad de las observaciones y conclusiones del presente análisis.

Finalmente, una de las formas de llamar la atención de los gerentes de salud y del público en general respecto de la información es publicándola, lo cual estimula su colección y la depuración de su calidad. Debe reconocerse que, aun con datos imperfectos, se pueden hacer análisis que faciliten la gestión en el campo de la salud a partir de una gran cantidad de información extraída de distintas fuentes secundarias en los países.

En síntesis, los SIG pueden ser utilizados para simplificar y sintetizar resultados complejos del análisis de la situación de salud. Además, su capacidad permite definir la magnitud y la distribución de los fenómenos de salud y sus factores determinantes; identificar desigualdades en materia de salud; identificar grupos de población que se encuentran en mayor riesgo de enfermar o morir; determinar la presencia de necesidades de salud insatisfechas; estratificar epidemiológicamente los grupos vulnerables de la población; determinar prioridades de salud e idear intervenciones focalizadas, y permitir que se programen y planeen actividades de salud con mayor eficacia y equidad. Esta tecnología está disponible, en diferentes formas y con distintos niveles de complejidad, para los usuarios de los servicios de salud pública, como se ha señalado en otras publicaciones (22). La OPS está facilitando su adopción mediante el desarrollo del paquete de programas computadorizados de SIG, denominado SIGEpi, diseñado específicamente con herramientas analíticas para el sector de la salud. SIGEpi se proporcionará gratuitamente como parte de la cooperación técnica con los países. 


\section{REFERENCIAS}

1. Organización Panamericana de la Salud. Gente sana en entornos saludables. Informe anual del Director, 1996. Washington, D.C.: OPS; 1997.

2. Organización Panamericana de la Salud. La situación de salud en la Región de las Américas. Informe anual del Director, 1998. Washington, D.C.: OPS; 1999.

3. Organización Panamericana de la Salud. La salud en las Américas. Edición 1998. Washington, D.C.: OPS; 1998. (Publicación Científica No. 569).

4. Organización Panamericana de la Salud, Programa Especial de Análisis de Salud. Iniciativa Regional de Datos Básicos en Salud. Sistema de Información Técnica en Salud. Washington, D.C.: OPS; 2001. [Sitio en Internet]. Se encuentra en: h t t p : / / w w w . p a h o.o r g / spanish/sha/coredata/tabulator/newTabula tor.htm. Acceso el 15 de julio de 2002.

5. Schneider MC, Castillo-Salgado C, Bacallao J, Loyola-Elizondo E, Mujica OJ, Vidaurre M, et al. Metodologías para la medición de desigualdades en salud. Rev Panam Salud Publica 2002; (Supl).

6. Duarte EC, Schneider MC, Souza RP, Ramalho W; Sardinha LMV, Barbosa da Silva $\mathrm{J}$, et al. Epidemiología das desigualdades en saúde no Brasil: um estudo exploratorio. Fundaçao Nacional de Saúde, Centro Nacional de Epidemiología y Organizaçao Pan-Americana da Saúde, Programa Especial de Análises de Saúde. Brasilia: CENEPI; 2002.

7. Organización Panamericana de la Salud. Situación de salud en las Américas. Indicadores básicos, 1996. Washington, D.C.: OPS; $1996 . \quad$ (Documento OPS/HDP/HDA.96.02).
8. Organización Panamericana de la Salud. Situación de salud en las Américas. Indicadores básicos, 1997. Washington, D.C. OPS; $1997 . \quad$ (Documento OPS/HDP/HDA.97.02)

9. Organización Panamericana de la Salud. Situación de Salud en las Américas. Indicadores básicos 1998. Washington, D.C.: OPS ; 1998.

(Documento OPS/HDP/HDA.98.02)

10. Organización Panamericana de la Salud. Situación de salud en las Américas. Indicadores básicos, 1999. Washington, D.C. OPS; 1999. (Documento OPS/SHA.99.01).

11. Organización Panamericana de la Salud. Situación de salud en las Américas. Indicadores básicos, 2000. Washington, D.C.: OPS; 2000. (Documento OPS/SHA.00.02).

12. Organización Panamericana de la Salud. El progreso en la salud de la población. Informe anual del Director, 2000. Washington, D.C.: OPS; 2000.

13. Ministerio de Salud de Brasil. Sistema único de saúde (DATASUS), indicadores de salud. [Sitio en Internet]. Se encuentra en: http:// tabnet.datasus.gov.br/tabnet/tabnet.htm. Acceso en noviembre de 2001 y abril de 2002.

14. Larrea C, Carrasco F, Cervantes J, Viedma N. INFOPLAN:.Atlas para la gestión local. Quito: Oficina de Planeación, Secretaría de la Presidencia; 1999.

15. Environmental Systems Research Institute. ESRI data and maps. Redlands: ESRI, Inc.; 2000.

16. Instituto Brasileño de Geografía y Estadística. Malha municipal digital, 1997. [CD-rom] Rio de Janeiro: IBGE; 1999.

17. Environmental Systems Research Institute. Digital chart of the world. Redlands: ESRI, Inc.; 2001.
18. SPSS. Statistical package for the social sciences for Windows. Release 10.05. [Software]. Chicago: SPSS Inc.; 1999.

19. Environmental Research Systems Institute. ArcView GIS 3.2. Redlands: ESRI Inc.; 1999.

20. Castillo-Salgado C. Estratificación epidemiológica de la malaria en las Américas. Bol Epidemiol (Organización Panamericana de la Salud) 1991;12:1-7.

21. Haynes R, Gale S. Deprivation and poor health in rural areas: inequalities hidden by averages. Health \& Place 2000;6:275-285.

22. Organización Panamericana de la Salud. Sistemas de información geográfica en salud. Conceptos básicos. Washington, D.C.: OPS; 2002.

23. López-Ríos O. Efecto de los servicios de salud y factores socioeconómicos en las diferencias espaciales de la mortalidad mexicana. Salud Publica Mex 1997;39:16-34.

24. Morgenstern H. Ecologic studies in epidemiology: concepts, principles and methods. Annu Rev Public Health 1995;16:61-81.

25. Pickle LW, Mungiole M, Jones GK, White AA. Atlas of the United States mortality. Hyattsville: National Center for Health Statistics; 1996.

Manuscrito recibido el 19 de julio de 2002. Aceptado para publicación, tras revisión, el 4 de octubre de 2002.

ABSTRACT Objective. To show how geographic information systems (GISs) can be used as technological tools to support health policy and public health actions.

Methods. We assessed the relationship between infant mortality and a number of socio-

Geographic information systems as a tool for monitoring health inequalities economic and geographic determinants. In explaining how GISs are applied, we stressed their ability to integrate data, which makes it possible to perform epidemiologic evaluations in a simpler, faster, automated way that simultaneously analyzes multiple variables with different levels of aggregation. In this study, GISs were applied in analyzing infant mortality data with three levels of aggregation in countries of the Americas from 1995 to 2000.

Results. Infant mortality in the Region of the Americas was estimated at an overall average of 24.4 deaths per 1000 live births. However, the inequalities that were found indicate that the probability of an infant death is almost 20 times greater in the less developed countries of the Region than in more developed ones. Mapping infant mortality throughout the Region of the Americas allowed us to identify the countries that need to focus more attention on health policy and health programs, but not to determine what specific actions are of the highest priority. An analysis of smaller geopolitical units (states and municipalities) revealed important differences within countries. This shows that, as is true of data for the entire Region of the Americas, using national-level average figures for indicators can obscure the differences that exist within countries. When we examined the relationship between female illiteracy and malnutrition as determinants of infant mortality in Brazil and Ecuador, we identified social and epidemiologic strata where risk factors had different distribution patterns and that thus require health interventions that match their individual social and epidemiologic profiles.

Conclusions. With this type of epidemiologic study using GISs at the local level of health services, it is easy to see how a health event and its risk factors behave at a specific period in time. It is also possible to identify patterns in the spatial distribution of risk factors and in these factors' potential impact on health. Using GISs in an appropriate way will make it easier to deliver more effective, equitable public health services. 